\title{
Sperm head abnormalities are associated with excessive omega- 6 fatty acids in two finch species feeding on sunflower seeds
}

Hanna Nyborg Støstad ${ }^{1 *}$, Melissah Rowe ${ }^{1,2}$, Arild Johnsen ${ }^{1}$, Oldřich Tomášek ${ }^{3,4}$, Tomáš Albrecht ${ }^{3}$, Jan Terje Lifjeld ${ }^{1}$

\author{
${ }^{1}$ Natural History Museum, University of Oslo, 0318 Oslo, Norway \\ ${ }^{2}$ Centre for Ecological and Evolutionary Synthesis, Dept. of Biosciences, University of Oslo, 0316 Oslo, \\ Norway \\ ${ }^{3}$ Institute of Vertebrate Biology, the Czech Academy of Sciences, 60365 Brno, Czechia \\ ${ }^{4}$ Charles University, Faculty of Science, 12844 Praha, Czechia \\ *Corresponding author. Email: h.n.stostad@nhm.uio.no
}

\begin{abstract}
Sunflower seeds are commonly provided in garden bird feeders, but contain high levels of linoleic acid (LA), an omega-6 ( $\omega-6)$ polyunsaturated fatty acid (PUFA). LA is associated with increased oxidative stress, which can damage cell membranes, and in particular sperm cells. We investigated the relationship between the proportion of LA in the blood (as well as the proportion of 10 other fatty acids, the proportion of total $\omega-6$ and $\omega-3$, and $\omega-6 / \omega-3$ ratio) and sperm quality (the frequency of sperm head abnormalities and sperm swimming speed) in two seed-eating finch species, greenfinches Chloris chloris and hawfinches Coccothraustes coccothraustes. The majority of birds were sampled in urban environments with direct access to sunflower seeds, whereas a smaller group were sampled in a rural area. We found that both species, but particularly greenfinches caught near feeders, exhibited levels of LA that were considerably higher than those reported for other wild birds in the literature, and higher than other bird species sampled at the same site. Furthermore, we found that the proportion of LA was positively correlated with the frequency of abnormal sperm heads (sperm missing the acrosome), while there was no significant effect of fatty acid composition on sperm swimming speed. Our results indicate that the sperm quality of finches may be negatively affected by a high intake of sunflower seeds, adding to a growing body of research showing that supplementary feeding can have detrimental side effects for urban animals. This is particularly relevant for the greenfinch, which is currently affected by disease and population declines.
\end{abstract}




\section{Introduction}

Bird feeders are a common feature of the urban environment, and have become increasingly important as a food source for urban birds. In the western world, it is estimated that around half of households provide food for wild birds in their gardens (Jones and Reynolds 2008; Davies et al. 2009), also during the breeding season (Orros \& Fellowes 2015). Such supplementary feeding may enhance bird survival and reproductive success (Robb et al. 2008; Fuller et al. 2012); however, there has been concern about potential negative effects, such as increased incidence of disease (Robb et al. 2008; Robinson et al. 2010). In addition to the potential disease transmission risks of aggregated birds at feeders, recent research shows that the nutritional composition of provided foods can negatively influence body condition and reproductive success of wild birds (Plummer et al. 2018). Households tend to provide foods that would not be found abundantly in the local environment, for example bread, peanuts, or sunflower seeds (Jones and Reynolds 2008; Støstad et al. 2017), and birds may not have evolved the capability to judge the nutritional value of such novel food items. Although urban birds select the most energy-rich human-based food types when offered a choice (Støstad et al. 2017), such foods are often high in calories yet inadequate in other vital aspects, e.g. vitamin content or fatty acid composition, and indiscriminate consumption could lead to malnutrition.

Polyunsaturated fatty acids (PUFAs) are fatty acids that are crucial for a number of important processes such as immune function and brain development (Patterson et al. 2012), but can also be detrimental when consumed in excess. In particular, a key dietary challenge is achieving a balanced ratio between omega-3 ( $\omega-3)$ and omega- 6 ( $\omega-6)$ PUFAs. Excessive amounts of $\omega-6$ PUFAs can lead to oxidative damage and increased inflammatory responses in both birds and humans (e.g. Larsson et al. 2004; Cherian 2007; Brown 2016). For example, linoleic acid (LA, 18:2n-6), one of the most abundant w-6 PUFAs in terrestrial plants (Hixson et al. 2015) and thus an important component in the diet of many animals, can be metabolised into reactive oxygen species such as hydrogen peroxide (Brown 2016). When such reactive oxygen species are not balanced by sufficient amounts of antioxidant enzymes, they cause oxidative stress, which damages cell membranes through lipid peroxidation. On the other hand, whilst excessive $\omega-6$ PUFAs can be detrimental, it is also important to ensure adequate intake of $\omega-3$ PUFAs. These $\omega$-3 PUFAs are essential for cell membrane structure and flexibility, and deficiency can cause poor growth, impaired vision and a number of other detrimental effects (Brenna and Carlson 2014; Twining et al. 2016a). Indeed, a high intake of dietary $\omega$-3 PUFAs has been shown to improve chick fitness in tree swallows (Twining et al. 2016b). Importantly, $\omega-3$ and $\omega-6$ PUFAs compete for digestive enzymes, which means that a higher intake of $\omega-3$ can decrease the amount of $\omega-6$ that is metabolised into reactive oxygen species (Brown 2016), emphasising the need for a balance between the two. 
Notably, many food types provided to wild birds by households are rich in $\omega-6$ PUFAs. In particular, sunflower seeds, which are commonly used in bird feeders, have a PUFA profile consisting almost exclusively of LA with very low levels of $\omega$-3 PUFAs (see Table 1). Thus, urban birds that use bird feeders extensively may have an unbalanced diet. Isaksson et al. (2017) found that urban house sparrows Passer domesticus had higher plasma levels of LA than rural birds, possibly due to access to different food sources, although they found the opposite pattern for great tits, indicating species differences in either diet or metabolism. Sparrows are predominantly seed eaters, as opposed to the more omnivorous great tits, so that sparrows may be more affected by varying availability of LA-rich seeds. In Norway, providing sunflower seeds for wild birds has become increasingly popular during the last few decades, as demonstrated by a gradual increase in the annual import of sunflower seeds intended for animal feeding from zero in 1993 to nearly 8 million $\mathrm{kg}$ in 2017, with an annual import value of 6 million USD in 2017 (Statistics Norway, 2018). Thus, investigating the physiological effects of feeding sunflower seeds to wild birds is highly relevant.

Avian sperm membranes are largely composed of $\omega-6$ PUFAs (Surai et al. 1998; Cerolini et al. 2003), so $\omega-6$ PUFAs are in fact essential to avian spermatogenesis for production of sperm membranes. However, $\omega-6$ PUFAs are at the same time potentially harmful to sperm quality through the production of reactive oxygen species as described above. The unsaturated fatty acids in sperm membranes are unstable and bond readily with reactive oxygen species (Gagnon et al. 1991), which oxidises the lipid membranes of the sperm, ultimately causing disruption of sperm functions (Aitken and Baker 2006, Bonisoli-Alquati et al. 2011). It is important that the membrane of the acrosome (the anterior part of the sperm head) remains intact until the point of fertilisation, as the enzymes contained within the acrosome are necessary for the acrosome reaction, allowing sperm to penetrate the ovum (Nishio and Matsuda 2017). The apparent contradiction between the beneficial and detrimental impacts of $\omega-6$ PUFAs means that a balanced intake of $\omega-6$ is essential to ensure optimal sperm quality in birds. Moreover, sperm quality may be particularly important for male fitness in many passerine bird species due to the high incidence of promiscuity and subsequent sperm competition (Griffith et al. 2002; Pizzari and Parker 2009), so that deficiencies or overconsumption of $\omega-6$ PUFAs could have considerable fitness consequences for these birds.

In captive bird populations, the effect of dietary PUFA content on sperm performance appears to be variable, and depends on age and levels of other nutrients such as vitamin $E$ (Cerolini et al. 2003; Khatibjoo et al. 2018). Diets enriched with $\omega$-3 PUFAs have been associated with increased fertility (Blesbois et al. 1997; Feng et al. 2015) and larger semen volume (Surai et al. 2000) compared to $\omega-6$ rich diets; although one study showed that an $\omega$-3-rich diet led to a slight decrease in sperm motility yet an increase in fertility (Kelso et al. 1997), and other studies have found no 
Table 1. Nutritional content of sunflower seeds. Data from USDA National Nutrient Database for Standard Reference, https://ndb.nal.usda.gov/ndb/. Note that the retailer of the provided sunflower seeds was unable to provide the exact nutrient content; additionally, nutritional content may vary slightly among sunflower varieties and batches. $C: D=$ number of carbon atoms:double bonds. SFA = saturated fatty acids, MUFA = mono-unsaturated fatty acids, PUFA = poly-unsaturated fatty acids.

\begin{tabular}{|c|c|c|c|}
\hline Trivial name (abbreviation) & C:D & Class & $\mathrm{g}($ of $100 \mathrm{~g})$ \\
\hline Palmitic acid & C16:0 & SFA & 2.21 \\
\hline Stearic acid & C18:0 & SFA & 1.69 \\
\hline Oleic acid & C18:1 n-9 & MUFA & 18.38 \\
\hline Linoleic acid (LA) & C18:2n-6 & $\omega-6$ PUFA & 23.05 \\
\hline Gamma-linolenic acid (GLA) & C18:3n-6 & $\omega-6$ PUFA & 0 \\
\hline Dihomo-gamma-linolenic acid (DGLA) & $C 20: 3 n-6$ & $\omega-6$ PUFA & 0 \\
\hline Arachidonic acid (ARA) & $C 20: 4 n-6$ & $\omega-6$ PUFA & 0 \\
\hline Alpha-linolenic acid (ALA) & $\mathrm{C} 18: 3 \mathrm{n}-3$ & $\omega-3$ PUFA & 0.06 \\
\hline Eicosapentaenoic acid (EPA) & $C 20: 5 n-3$ & $\omega-3$ PUFA & 0.01 \\
\hline Docosapentaenoic acid (DPA) & $C 22: 5 n-3$ & $\omega-3$ PUFA & 0 \\
\hline Docosahexaenoic acid (DHA) & $C 22: 6 n-3$ & $\omega-3$ PUFA & 0 \\
\hline Total $\omega-6$ PUFA & & & 23.05 \\
\hline Total $\omega$-3 PUFA & & & 0.07 \\
\hline Ratio $\omega-6 / \omega-3$ & & & 329.29 \\
\hline
\end{tabular}

difference in sperm motility (Cerolini et al. 2003; Khatibjoo et al. 2018). In comparison, research on fatty acid composition in the sperm of wild birds is scarce, and to our knowledge there are no studies on the impact of the nutritional content of the diets of wild birds on sperm quality. However, oxidative stress is negatively associated with sperm motility and swimming speed in wild great tits Parus major (Helfenstein et al. 2010) and barn swallows Hirundo rustica (Bonisoli-Alquati et al. 2011), and since an unbalanced fatty acid composition in the diet can aggravate oxidative stress, a diet rich in $\omega-6$ PUFAs could potentially be detrimental for sperm quality in wild birds.

Here, we first quantify the fatty acid composition in the blood of two seed-eating finch species, the greenfinch Chloris chloris and the hawfinch Coccothraustes coccothraustes, caught at sunflower seed feeders, and we compare these results to the fatty acid composition of a smaller number of individuals of six other common garden bird species. Secondly, we investigate the relationship between sperm performance and the proportion of LA in the blood of the two finch species; using birds that were caught in proximity to sunflower feeders as well as birds that were caught in rural, feeder-poor areas. We predict that seed-eating finches, especially those caught in proximity to feeders, will have higher blood levels of LA than other species, and that individuals with higher levels of LA will have lower sperm quality (as expressed by the proportion of abnormal sperm heads and sperm swimming speed). 


\section{Methods}

\section{Study species}

The two focal species of the study, greenfinches and hawfinches, were chosen mainly due to their feeding ecology as seed eaters with a preference for sunflower seeds. Personal observations as well as the literature (e.g. Brittingham and Temple 1992) suggest that both species, but particularly
Table 2. Sperm head abnormalities in greenfinches across four years of sampling. Samples from 2012 and 2013 are not included in further analyses.

\begin{tabular}{|c|ccc|}
\hline Year & $\begin{array}{c}\text { Number of } \\
\text { samples }\end{array}$ & $\begin{array}{c}\text { Percent } \\
\text { abnormalities }\end{array}$ & $\begin{array}{c}\text { Standard } \\
\text { deviation }\end{array}$ \\
\hline 2012 & 6 & 4.17 & 4.36 \\
\hline 2013 & 13 & 35.23 & 22.95 \\
\hline 2015 & 46 & 7.58 & 8.03 \\
\hline 2017 & 22 & 12.36 & 21.00 \\
\hline
\end{tabular}

greenfinches, are frequent visitors to sunflower feeders, and that they tend to include sunflowers as a substantial part of their diet (as opposed to e.g Parids (tits), which will also frequently visit feeders with other food types e.g. fats). Moreover, greenfinch populations are declining in the UK (Harris et al. 2018), in Norway (Falkenberg et al. 2018), and in Czechia (Reif et al. 2010), and the decline in the UK has been linked to disease spreading through their use of feeders (Robinson et al. 2010). As such, investigating further adverse effects from supplementary feeding on this species is particularly important, as it could help inform potential conservation actions. Finally, we have in the past observed remarkably high rates of abnormal sperm in the greenfinch (Table 2), especially in 2013 when several samples showed around 50\% sperm abnormalities (unfortunately we do not have fatty acid profiles for these older samples).

\section{Sampling}

For the current study, we sampled birds in two countries (Norway and Czechia) and across two years (2015 and 2017). We had variable catching success and there was variability in the quality of the collected data, i.e. some males for which we obtained fatty acid data had no sperm, and some had sufficient sperm for morphology assessment but not for sperm velocity assessment. We have aimed to maximise sample sizes in each analysis, rather than limiting analyses to the individuals for which we had all types of data; this means that sample sizes vary among the analyses.

The main part of the sampling was done in southeast Norway, in urban or suburban areas (see list of locations in Table S1), during the breeding season (April - June) of 2015 and 2017. At these sampling sites, we provided sunflower seeds in feeders throughout the preceding winter and up until (and including) the period of sampling. Sunflower seeds were from the Norwegian retailer Felleskjøpet, see nutrient content in Table 1. The purpose of the feeding was to maximise the chances of catching birds that had utilised sunflower feeders; however, we do not know to what extent individual birds used the feeders, and additionally, there is no guarantee that the sampled birds were overwintering residents at the sampling site. Still, our observations indicate that finches at 
the sampling sites used the feeders frequently and for long periods of time, so we consider it likely that sunflower seeds constitute a considerable part of the diet of the sampled birds.

At the feeder sites, we caught 63 greenfinches and 11 hawfinches across the two sampling years (Table S1). To compare the fatty acid profiles of these individuals with other local species, in particular species that tend to forage less at the sunflower feeders (based on personal observation and general species ecology), we sampled a small number of birds of other species from the feeder sites. These were eight siskins Spinus spinus, six chaffinches Fringilla coelebs, two great tits Parus major, two blue tits Cyanistes careuleus, two coal tits Periparus ater, and one goldfinch Carduelis carduelis. These were not used for sperm analysis. Additionally, three greenfinches and eight hawfinches were caught in rural areas in southern Czechia in 2015, with limited access to feeders (Tryjanowski et al. 2015). Sampling in an area with a low density of feeders as well as the areas with direct access to well-stocked feeders allowed us to catch birds that were likely to have ingested variable amounts of sunflower seeds, facilitating a correlational analysis between LA and sperm performance.

Birds were caught using mist nets. Blood samples were collected from the brachial vein, and a drop of blood was deposited on a blotting paper (Whatman 903 Protein Saver Card, Sigma-Aldrich) for subsequent fatty acid analyses. Sperm samples were taken using cloacal massage (Wolfson 1952; Kucera and Heidinger 2018), and upon collection all samples were fixed and stored in 5\% formaldehyde solution buffered with PBS. For a subset of samples (all greenfinches from 2015 from Norway) sperm were also used for velocity analysis, see below. All sampling was done in adherence with local guidelines for animal research and with all relevant permits. Birds were released immediately after sampling.

\section{Sperm morphology assessment}

Seven greenfinches (all from the Norwegian sites) had insufficient sperm and were used only for fatty acid analysis, and so in total 59 greenfinches and 19 hawfinches from both countries were used for sperm morphology assessment. To measure the proportion of morphologically abnormal sperm in a sample, we prepared sperm smears of the formaldehyde-fixed sperm. Sperm solution was smeared on a glass slide, air-dried, and rinsed with distilled water, before being assessed using a digital light microscope (DM6000 B, Leica Microsystems) set to 320x magnification. Images were taken with a digital camera (DFC420, Leica Microsystems, Heerbrugg, Switzerland) mounted on the microscope.

We aimed to assess at least 100 randomly selected sperm from each male, scoring each cell as having either 1) a morphologically normal sperm head, defined as a head with no obvious signs of damage and a shape that corresponded to the usual appearance for the species (fig. S1a); 2) a sperm 
that was missing the acrosome (fig. S1b); or 3) a misshapen sperm head that retained the acrosome but appeared abnormal in shape, for example had lost its helical appearance or was shaped like a hook (fig. S1c). In this study, we place slightly more emphasis on the category of missing acrosomes, as this is a well-defined sperm defect which is likely related to a premature acrosome reaction, whereas 'misshapen sperm heads' is a more vaguely defined trait and was also more difficult to assess accurately. All samples were assessed by the same person (HNS) who had no further information on the sample at the time of assessment.

\section{Sperm velocity assessment}

Sperm samples were collected as described above and immediately diluted in Phosphate Buffered Saline (PBS) pre-heated to $40^{\circ} \mathrm{C}$. Next, $6 \mu$ of the sperm suspension was loaded into a chamber slide (depth $20 \mu \mathrm{m}$, Leja ${ }^{\circledR}$, Netherlands) and sperm motion was recorded for six unique fields of view (5s each for a total of 30s) at $400 x$ using phase contrast microscopy (CX41, Olympus, Japan) connected to a digital camera (Legria HF S200, Canon, Japan). All samples, chamber slides, and the heated microscope stage (TP-S, Tokai Hit, Shizuoka, Japan) were maintained at a constant temperature of $40^{\circ} \mathrm{C}$ throughout.

Videos of sperm motion were analysed using computer-assisted sperm analysis (CASA; HTRCEROS sperm tracker, CEROS v.12, Hamilton Thorne Research) with frame rate set at $50 \mathrm{~Hz}$ and 25 frames (i.e. sperm cells were tracked for $0.5 \mathrm{~s}$ ) and quality control settings following Rowe et al. (2015). Each analysis was visually examined and cell detection parameters established to exclude non-sperm, contaminating particles: minimum contrast, 50-100; minimum cell size, 8-12 pixels; sperm head elongation (i.e. width/length), $<75 \%$. Additionally, we excluded non-continuous sperm tracks or sperm tracked for less than 10 frames, as well as tracks for which the maximum frame-toframe movement exceeded the average frame-to-frame movement by 4 SDs for the same track, as such tracks tend to represent tracking errors. Finally, to account for the effects of drift, tracks with an average path velocity (VAP) of $<35 \mu \mathrm{m} \mathrm{s}^{-1}$ or a straight line velocity (VSL) of $<25 \mu \mathrm{m} \mathrm{s}^{-1}$ were considered immotile. These cut-offs were based on an analysis (unpublished data) of dead sperm cells using the same recording and analysis conditions as those used in the current study. All CASA was done by the same person (MR) and without information about the male's identity.

Following this filtering process, we excluded samples with less than 5 motile sperm tracks from analyses of sperm swimming speed. After this exclusion, the total number of motile sperm tracked for each male ranged from 5 to 60 (median $=27$, mean $=29.08 \pm 19.28$ ), and the total number of samples with swimming speed data was 24 . For each sperm cell, we recorded cell straightline velocity (VSL, i.e. average velocity on a straight line between the start and endpoint of the sperm 
track), average path velocity (VAP, i.e. average velocity over a smoothed sperm track), and curvilinear velocity (VCL, i.e. velocity over the actual sperm track). VSL, VAP and VCL were all strongly correlated (Pearson's correlation, all $r>0.80$ ), and so we used VCL as an indication of sperm swimming speed.

\section{Fatty acid extraction and gas chromatography analysis}

Fatty acid analysis of blood samples was performed by Vitas AS (vitas.no), Norway. One punch of whole blood ( $6 \mathrm{~mm}$ diameter of blotting paper) was diluted with $900 \mu \mathrm{L} 0.5 \mathrm{M}$ sodium methylate. After methylation $\left(20 \mathrm{~min}, 450 \mathrm{rpm}, 50^{\circ} \mathrm{C}\right)$, fatty acid methyl esters (FAMEs) was extracted with 500 $\mu \mathrm{L}$ hexane and $300 \mu \mathrm{L}$ distilled water. After thorough mixing $(5 \mathrm{~min})$ and centrifugation $(5 \mathrm{~min}, 4000$ g), $3 \mu$ l of the aliquot was injected into a Gas Chromatography - Flame lonization Detector (GC-FID). GC-FID was performed with an Agilent 7890A Gas Chromatograph System (Agilent Technologies, Palo Alto, CA, USA). The fatty acids were separated on a Supelco SP2380 $(30 \mathrm{~m} \times 250 \mu \mathrm{m} \times 0.2 \mu \mathrm{m})$ column. The results are reported as area-\% of FAMEs.

\section{Statistical analysis}

All statistical analysis was done in R v 3.3.3 (R Core Team 2017). To analyse the relationship between the proportion of LA and sperm head abnormalities, we constructed two generalised linear mixed models (GLMM) using the glmmTMB package (Brooks et al. 2017), with the proportion of LA in the blood as a predictor variable, and either 1) the proportion of sperm with missing acrosomes or 2) the proportion of misshapen sperm heads as response variables. To account for the effects of species and sampling, we created a single compound variable which signified the country of collection, year of collection, and species (e.g. NO17GF), which was used as a random effect in all models. Note that using other combinations of random effects (e.g. only including 'year' and 'species', or using a nested random effect structure with year/country/species), produced similar results (not shown). Due to the non-normality of the sperm abnormality data (strong left skew, but not zero-inflated), we used a negative binomial distribution with a log link function, which provided better model fit than alternative model parameters. This was assessed using the plot(model) function in R (Q-Q plots and similar) as well as with the DHARMa package (Hartig 2017). The gImmTMB models also corrected for overdispersion.

Although the relationship between LA and sperm abnormalities was the main interest in the current study, we also present the relationship between 10 other measured fatty acids and sperm abnormalities, using similar GLMM models. The proportion of each fatty acid was the predictor variable in each model, with either the proportion of sperm with a missing acrosome or the proportion of misshapen sperm heads as a response variable. Finally we constructed three further GLMM models using 1 ) the total proportion of $\omega-6$ PUFAs, 2 ) the total proportion of $\omega-3$ PUFAs, and 
3) the $\omega-6 / \omega-3$ ratio as the predictor variables. These additional models were otherwise the same as the models with LA.

Three individuals (all hawfinches from Norway) showed particularly high levels of misshapen sperm heads (outside 4 times the interquartile range), and we suspect this was due to some unrelated factor such as disease. When these outliers were included in the models, this invalidated model assumptions based on visual analysis of the model plots. We therefore removed these individuals from the analyses. Results from models including the outliers are presented in Table S2.

To analyse the relationship between the proportion of LA and sperm swimming speed (in the subset of greenfinches from Norway from 2015), we used a linear mixed effect model (LME), with VCL for each motile sperm cell as a response variable, the individual proportion of LA as a predictor variable, and male ID as a random effect. We also constructed 13 similar LME models using the 10 other fatty acids, total $\omega-6$ PUFAs, total $\omega-3$ PUFAs, and the $\omega-6 / \omega-3$ ratio as predictors.

\section{Results}

Linoleic acid (LA, 18:2n-6) was the most abundant fatty acid in the blood of both greenfinches and hawfinches, as it accounted for $36.2 \%$ of all fatty acids in the greenfinch (Fig. 1a) and $28.3 \%$ in the hawfinch (Fig. 1b). The proportion of LA was higher in these two species compared to other sampled species (Fig. 2), although the chaffinches also exhibited relatively high proportions of LA. Nearly half of total fatty acids in the greenfinch were $\omega-6$ PUFAs (46.2\%), versus $33.5 \%$ in the hawfinch. In

a)

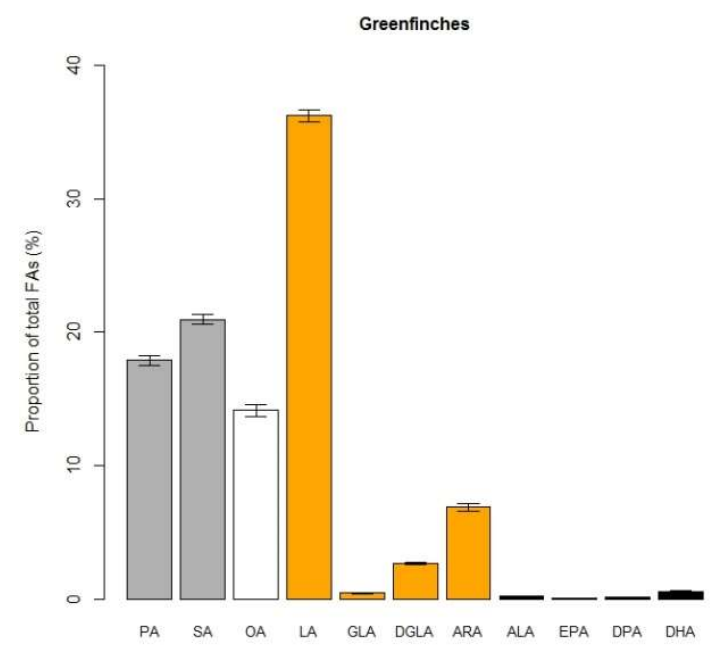

b)

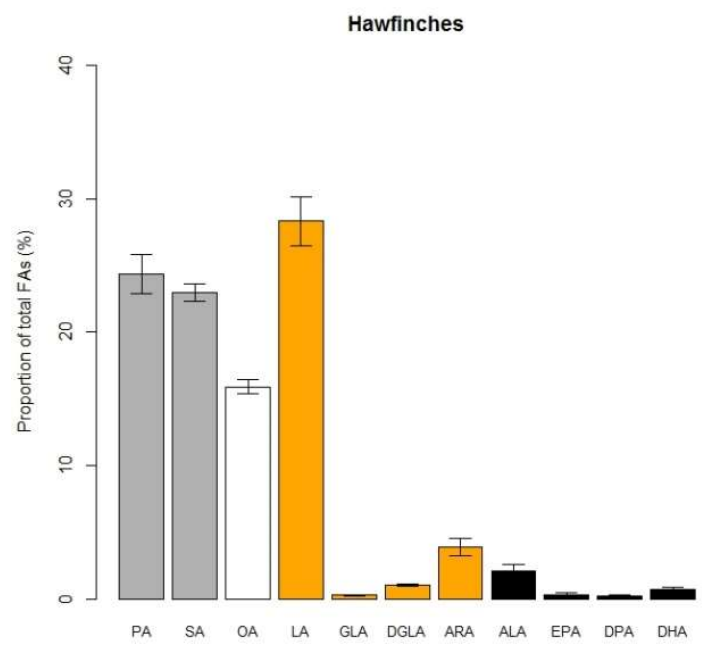

Figure 1. Proportion of fatty acids (\% of total fatty acid content) in a) greenfinches ( $n=66)$ and b) hawfinches $(n=19)$. Light grey bars are saturated fatty acids (SFA), white bars are mono-unsaturated fatty acids (MFAs), orange bars are $\omega-6$ poly-unsaturated fatty acids (PUFAs), and black bars are $\omega-3$ PUFAs. Error bars are $+/-1$ standard error. PA $=$ Palmitic acid, SA = Stearic acid, OA = Oleic acid, LA = Linoleic acid, GLA = Gamma-linolenic acid, DGLA = Dihomo-gamma-linolenic acid, ARA = Arachidonic acid, ALA = Alpha-linolenic acid, EPA = Eicosapentaenoic acid, DPA = Docosapentaenoic acid, DHA = Docosahexaenoic acid. 
contrast, $\omega-3$ PUFAs accounted for only $0.9 \%$ of total FAs in the greenfinch and $3.3 \%$ in the hawfinch. The mean $\omega-6 / \omega-3$ ratio (the mean of all individual $\omega-6 / \omega-3$ ratios) was 75.7 for the greenfinch and 29.4 for the hawfinch.

The proportion of LA was lower in birds caught in a feeder-poor area in Czechia than in birds caught in proximity to sunflower feeders in Norway ( $23.6 \%$ versus $36.3 \%$ respectively, Fig. 3 ), and the total proportion of $\omega-6$ PUFAs was also lower in the Czech birds (27.7\% versus $46.1 \%)$. Conversely, the total proportion of $\omega-3$ PUFAs was higher in Czech birds than in Norwegian birds (4.1\% versus $0.9 \%)$. For all mean fatty acid values, see table S3 (all greenfinches and hawfinches used for analysis) and S4 (all eight species sampled in Norway in 2015).

The percentage of sperm with a missing acrosome varied from $0 \%$ to $32 \%$ (mean: $6.1 \%$ ) in individual males, with higher mean rates in greenfinch than in hawfinch males $(6.5 \%$ versus $4.8 \%$ respectively), and lower rates in 2015 than in 2017 (5.7\% versus 7.4\%). The proportion of sperm with a missing acrosome was positively correlated with the proportion of LA in the blood (Table 3, Fig. 4) and with the total proportion of $\omega-6$ PUFAs (Table 3). Additionally, there was a tendency towards a negative correlation with eicosapentaenoic acid (EPA, $p=0.067$, Table 3). Notably, all correlations between sperm with a missing acrosome and $\omega-6$ PUFAs were positive, whereas all correlations with $\omega-3$ PUFAs were negative.

The proportion of sperm with a misshapen sperm head varied from $0 \%$ to $15 \%$ (excluding outliers; range incl. outliers $0 \%-72 \%)$. The proportion of sperm with misshapen heads was not related to the proportion of LA in the blood (Table 4), while there was a positive correlation between sperm with misshapen heads and gamma-linolenic acid (GLA, Table 4).

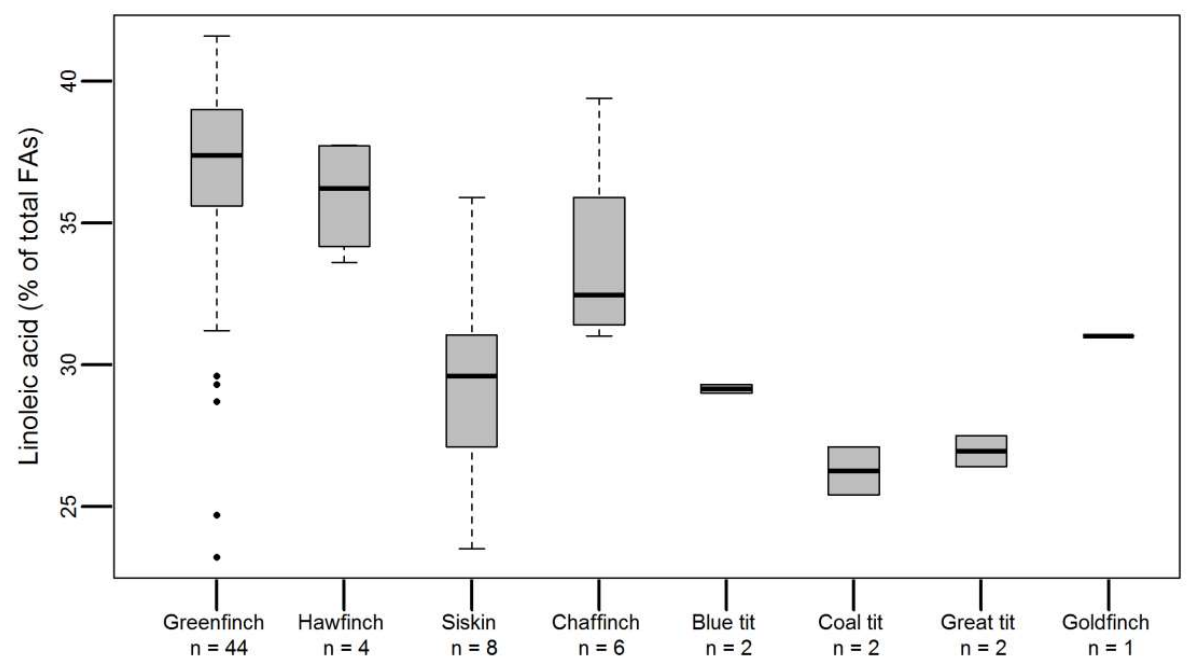

Figure 2. The level of linoleic acid (C18:2n-6, \% of total fatty acids) in the blood of eight bird species caught near sunflower feeders in Norway in 2015. 
Table 3. Generalised linear mixed models showing the relationship between the proportion of fatty acids in the blood and the proportion of sperm with a missing acrosome, in 59 greenfinches and 19 hawfinches. A single variable indicating the year, species and the country of collection was included as a random effect in all models. C:D = number of carbon atoms:double bonds, $\mathrm{LCL}=$ lower confidence limit, $\mathrm{UCL}=$ upper confidence limit. Significant results are presented in bold.

\begin{tabular}{|c|c|c|c|c|c|c|c|}
\hline Predictors & C:D & Response & df & $z$ & $p$ & $r$ & LCL, UCL \\
\hline Palmitic acid & C16:0 & Sperm with no acrosome & 74 & -1.616 & 0.106 & -0.18 & $-0.38,0.04$ \\
\hline Stearic acid & C18:0 & Sperm with no acrosome & 74 & -1.625 & 0.104 & -0.19 & $-0.39,0.04$ \\
\hline Oleic acid & C18:1 n-9 & Sperm with no acrosome & 74 & -1.127 & 0.260 & -0.13 & $-0.34,0.10$ \\
\hline Linoleic acid (LA) & C18:2 n-6 & Sperm with no acrosome & 74 & 2.491 & 0.013 & 0.28 & $0.06,0.46$ \\
\hline Gamma-linolenic acid (GLA) & C18:3n-6 & Sperm with no acrosome & 74 & 0.211 & 0.833 & 0.02 & $-0.20,0.24$ \\
\hline $\begin{array}{l}\text { Dihomo-gamma-linolenic acid } \\
\text { (DGLA) }\end{array}$ & $C 20: 3 n-6$ & Sperm with no acrosome & 74 & 0.625 & 0.532 & 0.07 & $-0.15,0.29$ \\
\hline Arachidonic acid (ARA) & $C 20: 4 n-6$ & Sperm with no acrosome & 74 & 1.433 & 0.152 & 0.16 & $-0.06,0.37$ \\
\hline Alpha-linolenic acid (ALA) & $\mathrm{C} 18: 3 \mathrm{n}-3$ & Sperm with no acrosome & 74 & -1.615 & 0.106 & -0.18 & $-0.38,0.04$ \\
\hline Eicosapentaenoic acid (EPA) & $C 20: 5 n-3$ & Sperm with no acrosome & 74 & -1.833 & 0.067 & -0.21 & $-0.40,0.02$ \\
\hline Docosapentaenoic acid (DPA) & $C 22: 5 n-3$ & Sperm with no acrosome & 74 & -1.433 & 0.152 & -0.16 & $-0.37,0.06$ \\
\hline Docosahexaenoic acid (DHA) & $C 22: 6 n-3$ & Sperm with no acrosome & 74 & -0.588 & 0.557 & -0.07 & $-0.28,0.16$ \\
\hline Total $\omega-6$ PUFA & & Sperm with no acrosome & 74 & 2.275 & 0.023 & 0.26 & $0.03,0.44$ \\
\hline Total $\omega$-3 PUFA & & Sperm with no acrosome & 74 & -1.719 & 0.086 & -0.20 & $-0.40,0.03$ \\
\hline Ratio $\omega-6 / \omega-3$ & & Sperm with no acrosome & 74 & 0.532 & 0.594 & 0.06 & $-0.16,0.28$ \\
\hline
\end{tabular}

Table 4. Generalised linear mixed models showing the relationship between the proportion of fatty acids in the blood and the proportion of sperm with a misshapen/abnormal head, in 59 greenfinches and 16 hawfinches (excluding three outliers). A single variable indicating the year, species and the country of collection was included as a random effect in all models. C:D = number of carbon atoms:double bonds, $\mathrm{LCL}=$ lower confidence limit, $\mathrm{UCL}=$ upper confidence limit. Significant results are presented in bold.

\begin{tabular}{|lllccccc|}
\hline Predictors & C:D & Response & df & z & $\mathbf{p}$ & $\boldsymbol{r}$ & LCL, UCL \\
\hline Palmitic acid & C16:0 & Misshapen sperm heads & 71 & -0.363 & 0.717 & -0.04 & $-0.27,0.19$ \\
\hline Stearic acid & C18:0 & Misshapen sperm heads & 71 & 0.297 & 0.766 & 0.04 & $-0.19,0.26$ \\
\hline Oleic acid & C18:1 n-9 & Misshapen sperm heads & 71 & 1.460 & 0.144 & 0.17 & $-0.06,0.38$ \\
\hline Linoleic acid (LA) & C18:2 n-6 & Misshapen sperm heads & 71 & -0.698 & 0.485 & -0.08 & $-0.30,0.15$ \\
\hline Gamma-linolenic acid (GLA) & C18:3 n-6 & Misshapen sperm heads & $\mathbf{7 1}$ & $\mathbf{- 2 . 2 1 9}$ & $\mathbf{0 . 0 2 7}$ & $-\mathbf{0 . 2 5}$ & $-\mathbf{0 . 4 5 , - 0 . 0 3}$ \\
\hline $\begin{array}{l}\text { Dihomo-gamma-linolenic acid } \\
\text { (DGLA) }\end{array}$ & C20:3 n-6 & Misshapen sperm heads & 71 & 1.035 & 0.301 & 0.12 & $-0.11,0.34$ \\
\hline Arachidonic acid (ARA) & C20:4 n-6 & Misshapen sperm heads & 71 & -0.352 & 0.725 & -0.04 & $-0.26,0.19$ \\
\hline Alpha-linolenic acid (ALA) & C18:3 n-3 & Misshapen sperm heads & 71 & -0.281 & 0.779 & -0.03 & $-0.26,0.20$ \\
\hline Eicosapentaenoic acid (EPA) & C20:5 n-3 & Misshapen sperm heads & 71 & -0.784 & 0.433 & -0.09 & $-0.31,0.14$ \\
\hline Docosapentaenoic acid (DPA) & C22:5 n-3 & Misshapen sperm heads & 71 & -0.682 & 0.495 & -0.08 & $-0.30,0.15$ \\
\hline Docosahexaenoic acid (DHA) & C22:6 n-3 & Misshapen sperm heads & 71 & -0.662 & 0.508 & -0.08 & $-0.30,0.15$ \\
\hline Total $\omega$-6 PUFA & & Misshapen sperm heads & 71 & -0.523 & 0.601 & -0.06 & $-0.28,0.17$ \\
\hline Total $\omega$-3 PUFA & & Misshapen sperm heads & 71 & -0.552 & 0.581 & -0.07 & $-0.29,0.17$ \\
\hline Ratio $\omega$-6 $/ \omega$-3 & & Misshapen sperm heads & 71 & 0.263 & 0.792 & 0.03 & $-0.20,0.26$ \\
\hline
\end{tabular}




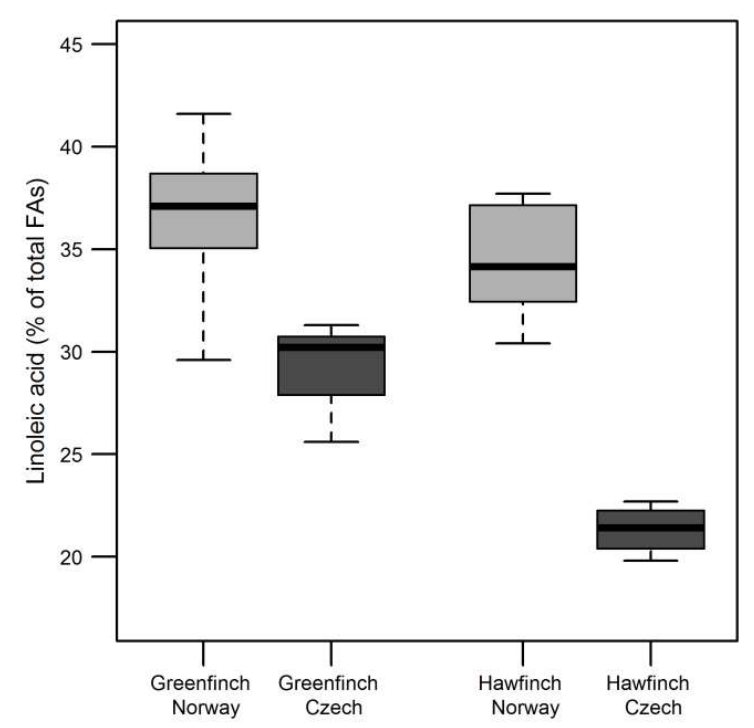

Figure 3. The level of linoleic acid (C18:2n-6, \% of total fatty acids) in the blood of greenfinches from Norway $(n=63)$ and Czechia $(n=3)$, and hawfinches from Norway $(n=11)$ and Czechia $(n=8)$.

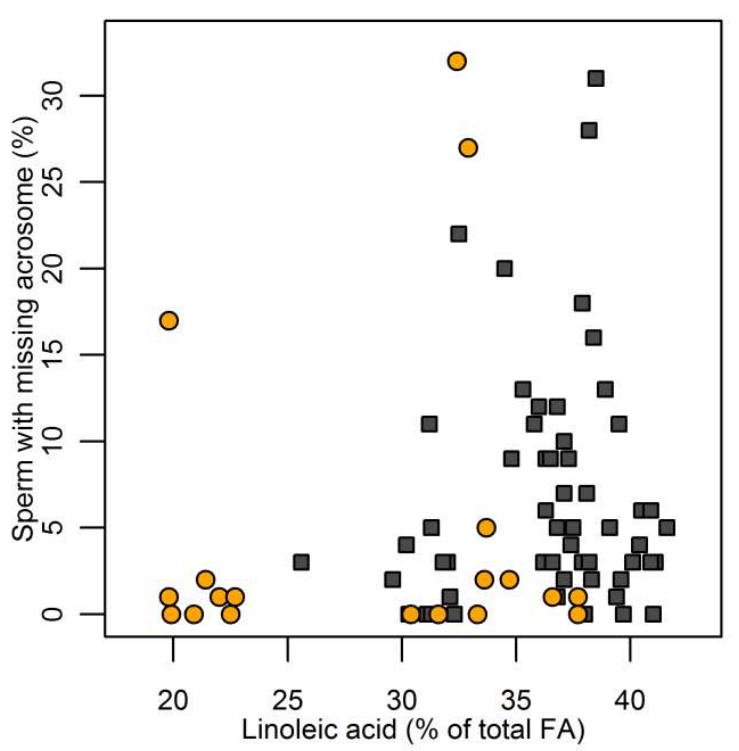

Figure 4. The relationship between the proportion of sperm with a missing acrosome (\% of 100 sperm) and the proportion of linoleic acid (C18:2n-6, \% of total fatty acids) in the blood of 58 greenfinches (dark grey squares) and 15 hawfinches (orange circles).

\section{Discussion}

144 We found that LA, a $\omega$-6 PUFA, was the most abundant fatty acid in the blood of greenfinches and hawfinches caught near sunflower feeders. In contrast, $\omega$-3 PUFAs constituted a small proportion of the total fatty acids, in particular for the greenfinch, where the $\omega-6 / \omega-3$ ratio exceeded $75: 1$. There was a positive correlation between the proportion of LA and the frequency of sperm with a missing acrosome, suggesting a detrimental effect of LA-rich diets on reproductive health in wild finches, although we found little evidence of an effect of LA on the frequency of misshapen sperm or on sperm swimming speed.

LA constituted around a third of the total fatty acids in the blood of both greenfinches and hawfinches, with particularly high levels for greenfinches sampled at bird feeders (36.6\%). This is relatively high compared to values reported in the literature for wild great tits (14-20\% depending on habitat and season, Andersson et al. 2015; Isaksson et al. 2015; Isaksson et al. 2017), as well as for blue tits, tree sparrows, and house sparrows (10-20\%, Isaksson et al. 2017), and for humans (23.5\%, Grimsgaard et al. 1999), although those data are from plasma rather than full blood. Similarly, the levels of LA we found in the greenfinches and hawfinches were higher than for the six other sampled species; in particular higher than the Parids (tits). Finches prefer to specifically visit sunflower feeders, whereas Parids tend to consume many types of provided foods, such as fats. Moreover, birds from feeder-dense areas in Norway had higher levels of LA than Czech birds, and the differences between 
countries were larger than differences between species within a country. These results indicate that fatty acid profiles are relatively labile, and are easily affected by food composition. This is interesting in terms of comparative studies. Furthermore, LA is an essential fatty acid, meaning it must be consumed through the diet and cannot be synthesised through metabolism. Together, these results support the hypothesis that a sunflower-rich diet influences the fatty acid composition of the finches' blood.

We found that the level of LA in the blood was positively correlated with the frequency of sperm that were missing the acrosome. This relationship could potentially be related to oxidative stress. As described in the introduction, LA can be metabolised into hydrogen peroxide, a reactive oxygen species, which easily reacts with the unstable bonds of the unsaturated fatty acids in the sperm membrane, causing sperm dysfunction (Aitken and Baker 2006; Brown 2016). Indeed, damage of the acrosomal membrane can lead to premature acrosome reactions, and oxidative stress has been shown to lead to premature acrosome reactions in rat sperm (Hsu et al. 1998). Thus, there is a possibility that the sperm we observed had undergone premature acrosome reactions due to damaged acrosomal membranes. Indeed, greenfinches have strongly helical sperm heads with wide, exposed acrosomal membranes, and this type of cell morphology is associated with increased frequency of abnormal sperm (Støstad et al. 2018a). Thus, it appears that the sperm of finches feeding on LA-rich diets might be affected by oxidative stress. Tomášek et al. (2017) found that oxidative challenge did not affect the number of abnormal sperm in zebra finches Taeniopygia guttata, but they included sperm with tail deformities in their data, which may show different responses than sperm heads to oxidative stress, and they also used diquat dibromide in the drinking water rather than changing dietary fatty acid composition. Moreover, our results also show a tendency for a negative correlation between EPA, a $\omega-3$ PUFA, and the frequency of sperm with no acrosome, which is consistent with previous literature; $\omega$-3 PUFAs such as alpha-linolenic acid (ALA, 18:3n-3) have been shown to be associated with increased fertility in cockerels (Kelso et al. 1997) and improved sperm motility in boars (Liu et al. 2017). Our results therefore indicate that excess amounts $\omega-6$ PUFAs, and possibly insufficient amounts of $\omega-3$ PUFAs, could be detrimental for sperm head integrity.

Intriguingly, we found a negative correlation between GLA, a $\omega-6$ PUFA, and the proportion of misshapen sperm heads. To our knowledge, there are no reports of any particular association between avian (or human) sperm and GLA in the literature, and there is no measureable quantity of GLA in sunflower seeds, so we cannot be certain of the cause of this correlation. However, the proportion of GLA in our samples was relatively low, and sperm consist of both $\omega-3$ and $\omega-6$ PUFAs (Cerolini et al. 2003); a deficiency of a necessary $\omega-6$ PUFA can also potentially be detrimental for 
sperm quality. As the fatty acid data are relative values rather than absolute values, there is also a possibility that differences in the proportions of particular fatty acids in the blood was due to variable amounts of other fatty acids, and/or that particular fatty acids had been deposited disproportionately into other tissues. For example, low levels of GLA in the blood could mean high levels of GLA in sperm or sperm-producing tissues (note that this is also the case for LA). For this reason, future research could aim to also test the fatty acid composition of the sperm. There is also the possibility that the correlation is statistically significant simply by chance, or related to an unknown third factor.

The recommended dietary $\omega-6 / \omega-3$ ratio for optimal fertility rate has been reported to about 6:1 to 9:1 for cockerels (Zanini et al. 2003). In contrast, the $\omega-6 / \omega-3$ ratio of sunflower seeds is 329:1, which far exceeds this recommendation. This means that a diet consisting solely of sunflower seeds is unlikely to be optimal for reproductive function in wild birds, and indeed, the levels of sperm abnormality we observed exceed levels that have been shown to negatively impact fertility in broiler chickens (Wilson et al. 1979). Birds require several sperm for successful embryo development (polyspermy, Hemmings and Birkhead 2015), and especially in a situation of intense sperm competition, males are likely to experience a decrease in paternity success if the number of normal sperm declines. Thus, there could be selection pressure for males to avoid over-consumption of food items with excessive $\omega-6$ PUFAs, such as sunflower seeds.

Interestingly, sunflower seeds are rich in vitamin E, which is considered to be an effective antioxidant, and supplementation with antioxidants has been shown to lead to fewer sperm abnormalities in zebra finches (Tomášek et al. 2017). However, our results indicate that the antioxidants in sunflower seeds are not sufficient to counteract the negative influence of the unbalanced $\omega-6 / \omega-3$ ratio. Moreover, as excessive $\omega-6$ PUFAs can increase inflammatory responses (Cherian 2007), over-consumption may be detrimental to the general health status of individuals, particularly for birds in challenging conditions, e.g. birds affected by disease. This is especially relevant for greenfinches due to the current prevalence of the parasite Trichomonas gallinae (Robinson et al. 2010) as well as the greenfinches' susceptibility to Salmonella infections (Refsum et al. 2003; Grant et al. 2007). Considering that the average $\omega-6 / \omega-3$ ratio in the blood of greenfinches in our study was $76: 1$, it is possible that such high levels of $\omega-6$ could aggravate the already poor health condition of infected birds.

As we did not observe a statistically significant correlation between the proportion of any fatty acid and sperm swimming speed, there appears to be little effect of fatty acid composition on the functioning of the morphologically normal sperm. Alternatively, the abnormal sperm are still able 
to swim normally, but given the correlation between sperm head shape and swimming speed reported for songbirds (Støstad et al. 2018b), this is perhaps less likely. Unfortunately, we were not able to acquire robust data for the proportion of motile sperm due to poor video/sample quality, but previous studies show no association between fatty acid composition and sperm motility in poultry (Khatibjoo et al. 2018) or in men (Conquer et al. 2000).

In summary, we demonstrate that urban finches have high blood levels of LA (a $\omega-6$ PUFA), possibly caused by consumption of LA-rich sunflower seeds. Our results also indicate that such high levels of LA may be detrimental for sperm quality in these finches. We propose that the mechanism could be membrane damage due to oxidative stress and subsequent premature acrosome reactions; however, we encourage further research in this area to experimentally assess a causal relationship between a sunflower-rich diet and sperm quality, as well as exploring the physiological mechanisms. Nevertheless, our results provide early indications that a uniform diet of sunflower seeds may be detrimental for the reproductive health of finches. If this proves to be the case, conservation managers should consider whether the rising popularity of bird feeding might be contributing to the decline of greenfinch populations.

\section{References}

Aitken, R. J., Baker, M. A. (2006). Oxidative stress, sperm survival and fertility control. Molecular and Cellular Endocrinology, 250: 66-69.

Andersson, M. N., Wang, H.-L., Nord, A., Salmón, P., Isaksson, C. (2015). Composition of physiologically important fatty acids in great tits differs between urban and rural populations on a seasonal basis. Frontiers in Ecology and Evolution, 3: 93.

Blesbois, E., Lessire, M., Grasseau, I., Hallouis, J., Hermier, D. (1997). Effect of dietary fat on the fatty acid composition and fertilizing ability of fowl semen. Biology of Reproduction, 56: 1216-1220.

Bonisoli-Alquati, A., Møller, A. P., Rudolfsen, G., Saino, N., Caprioli, M., Ostermiller, S., Mousseau, T. A. (2011). The effects of radiation on sperm swimming behavior depend on plasma oxidative status in the barn swallow (Hirundo rustica). Comparative Biochemistry and Physiology Part A: Molecular \& Integrative Physiology, 159: 105-112.

Brenna, J. T., Carlson, S. E. (2014). Docosahexaenoic acid and human brain development: evidence that a dietary supply is needed for optimal development. Journal of Human Evolution, 77: 99-106.

Brittingham, M. C., Temple, S. A. (1992). Does winter bird feeding promote dependency? Journal of Field Ornithology, 63: 190-194.

Brooks, M. E., Kristensen, K., van Benthem, K. J., Magnusson, A., Berg, C. W., Nielsen, A., Skaug, H.J., Mächler, M., Bolker, B. M. (2017). glmmTMB balances speed and flexibility among packages for zero-inflated generalized linear mixed modeling. The $R$ Journal, 9: 378-400.

Brown, R. A. (2016). Bioactive oxidised products of omega- 6 and omega-3, excess oxidative stress, oxidised dietary intake and antioxidant nutrient deficiencies, in the context of a modern diet. In M. V. Hegde, A. A. Zanwar, \& S. P. Adekar (Eds.), Omega-3 Fatty Acids: Keys to Nutritional Health (pp. 349-383). Cham: Springer International Publishing.

Cerolini, S., Pizzi, F., Gliozzi, T., Maldjian, A., Zaniboni, L., Parodi, L. (2003). Lipid manipulation of chicken semen by dietary means and its relation to fertility: a review. World's Poultry Science Journal, 59: 65-75.

Cherian, G. (2007). Metabolic and cardiovascular diseases in poultry: role of dietary lipids. Poultry Science, 86: 1012-1016. 
Conquer, J. A., Martin, J. B., Tummon, I., Watson, L., Tekpetey, F. (2000). Effect of DHA supplementation on DHA status and sperm motility in asthenozoospermic males. Lipids, 35: 149-154.

Davies, Z. G., Fuller, R. A., Loram, A., Irvine, K. N., Sims, V., Gaston, K. J. (2009). A national scale inventory of resource provision for biodiversity within domestic gardens. Biological Conservation, 142: 761-771.

Falkenberg, F., Berland, O. J., Ree, M. (2018). Hagefugltellingen 2018. Retrieved from http://www.birdlife.no/organisasjonen/nyheter/?id=2018

Feng, Y., Ding, Y., Liu, J., Tian, Y., Yang, Y., Guan, S., Zhang, C. (2015). Effects of dietary omega-3/omega-6 fatty acid ratios on reproduction in the young breeder rooster. BMC Veterinary Research, 11: 73.

Fuller, R. A., Irvine, K. N., Davies, Z. G., Armsworth, P. R., Gaston, K. J. (2012). Interactions between people and birds in urban landscapes. In: Lepczyk, C.A. and Warren, P.S. (Eds) Urban bird ecology and conservation, Studies in Avian Biology, No. 45, pp. 249-266.

Gagnon, C., Iwasaki, A., Lamirande, E., Kovalski, N. (1991). Reactive oxygen species and human spermatozoa. Annals of the New York Academy of Sciences, 637: 436-444.

Grant, D., Todd, P. A., Pennycott, T. (2007). Monitoring wild greenfinch (Carduelis chloris) for Salmonella enterica typhimurium. Ecological research, 22: 571-574.

Griffith, S. C., Owens, I. P. F., Thuman, K. A. (2002). Extra pair paternity in birds: a review of interspecific variation and adaptive function. Molecular Ecology, 11: 2195-2212.

Grimsgaard, S., Bønaa, K. H., Jacobsen, B. K., Bjerve, K. S. (1999). Plasma saturated and linoleic fatty acids are independently associated with blood pressure. Hypertension, 34: 478-483.

Harris, S. J., Massimino, D., Gillings, S., Eaton, M. A., Noble, D. G., Balmer, D. E., Procter, D., Pearce-Higgins, J.W., Woodcock, P. (2018). The Breeding Bird Survey 2017. BTO Research Report 706, British Trust for Ornithology.

Hartig, F. (2017). DHARMa: residual diagnostics for hierarchical (multi-level/mixed) regression models. R package version 0.1. 5 .

Helfenstein, F., Losdat, S., Møller, A. P., Blount, J. D., Richner, H. (2010). Sperm of colourful males are better protected against oxidative stress. Ecology Letters, 13: 213-222.

Hemmings, N., Birkhead, T. (2015). Polyspermy in birds: sperm numbers and embryo survival. Proceedings of the Royal Society B, 282: 20151682.

Hixson, S. M., Sharma, B., Kainz, M. J., Wacker, A., Arts, M. T. (2015). Production, distribution, and abundance of long-chain omega-3 polyunsaturated fatty acids: a fundamental dichotomy between freshwater and terrestrial ecosystems. Environmental Reviews, 23: 414-424.

Hsu, P.-C. H. C.-C., Chen, M.-Y. L. L.-Y., Guo, Y. L. (1998). Lead-induced changes in spermatozoa function and metabolism. Journal of Toxicology and Environmental Health Part A, 55: 45-64.

Isaksson, C., Hanson, M. A., Burdge, G. C. (2015). The effects of spatial and temporal ecological variation on fatty acid compositions of wild great tits Parus major. Journal of Avian Biology, 46: 245-253.

Isaksson, C., Andersson, M. N., Nord, A., von Post, M., Wang, H.-L. (2017). Species-dependent effects of the urban environment on fatty acid composition and oxidative stress in birds. Frontiers in Ecology and Evolution, 5: 44.

Jones, D. N., Reynolds, S. J. (2008). Feeding birds in our towns and cities: a global research opportunity. Journal of Avian Biology, 39: 265-271.

Kelso, K., Cerolini, S., Speake, B., Cavalchini, L., Noble, R. (1997). Effects of dietary supplementation with $\alpha-$ linolenic acid on the phospholipid fatty acid composition and quality of spermatozoa in cockerel from 24 to 72 weeks of age. Journal of Reproduction and Fertility, 110: 53-59.

Khatibjoo, A., Kermanshahi, H., Golian, A., Zaghari, M. (2018). The effect of n-6/n-3 fatty acid ratios on broiler breeder performance, hatchability, fatty acid profile and reproduction. Journal of Animal Physiology and Animal Nutrition. Early view ahead of print; doi: 10.1111/jpn.12904.

Kucera, A., Heidinger, B. (2018). Avian semen collection by cloacal massage and isolation of DNA from sperm. J. Vis. Exp., DOI: 10.3791/55324.

Larsson, S. C., Kumlin, M., Ingelman-Sundberg, M., Wolk, A. (2004). Dietary long-chain n- 3 fatty acids for the prevention of cancer: a review of potential mechanisms. The American Journal of Clinical Nutrition, 79 : 935-945.

Liu, Q., Zhou, Y.-F., Duan, R.-J., Wei, H.-K., Peng, J., Jiang, S.-W. (2017). Dietary n-6: n-3 ratio and Vitamin E improve motility characteristics in association with membrane properties of boar spermatozoa. Asian Journal of Andrology, 19: 223.

Nishio, S., T. Matsuda. 2017. Fertilization 1: Sperm-egg interaction. In T., Sasanami, ed. Avian reproduction. Springer, Singapore. 
Orros, M.E., Fellowes, M.D. (2015). Wild bird feeding in an urban area: intensity, economics and numbers of individuals supported. Acta ornithologica, 50: 43-58.

Patterson, E., Wall, R., Fitzgerald, G., Ross, R., Stanton, C. (2012). Health implications of high dietary omega-6 polyunsaturated fatty acids. Journal of Nutrition and Metabolism, 2012: 539426.

Pizzari, T., Parker, G. A. (2009). Sperm competition and sperm phenotype. In T. R. Birkhead, D. J. Hosken, \& S. Pitnick (Eds.), Sperm biology: an evolutionary perspective (pp. 207-245). Amsterdam: Academic Press.

Plummer, K. E., Bearhop, S., Leech, D. I., Chamberlain, D. E., Blount, J. D. (2018). Effects of winter food provisioning on the phenotypes of breeding blue tits. Ecology and Evolution, 8: 5059-5068.

R Core Team. (2017). R: A language and environment for statistical computing. Vienna, Austria: R Foundation for Statistical Computing. http://www.R-project.org/

Refsum, T., Vikøren, T., Handeland, K., Kapperud, G., Holstad, G. (2003). Epidemiologic and pathologic aspects of Salmonella typhimurium infection in passerine birds in Norway. Journal of Wildlife Diseases, 39: 6472.

REIF, J., VERMOUZEK, Z., VOŘíŠEK, P., ŠŤASTNÝ, K., BEJČEK, V., \& FLOUSEK, J. (2010). Population changes in Czech passerines are predicted by their life-history and ecological traits. Ibis, 152: 610-621.

Robb, G. N., McDonald, R. A., Chamberlain, D. E., Bearhop, S. (2008). Food for thought: supplementary feeding as a driver of ecological change in avian populations. Frontiers in Ecology and the Environment, 6: 476484.

Robinson, R. A., Lawson, B., Toms, M. P., Peck, K. M., Kirkwood, J. K., Chantrey, J., Clatworthy, Innes R., Evans, A.D., Hughes, L.A., Hutchinson, O. C. (2010). Emerging infectious disease leads to rapid population declines of common British birds. Plos One, 5: e12215.

Rowe, M., Griffith, S. C., Hofgaard, A., Lifjeld, J. T. (2015). Subspecific variation in sperm morphology and performance in the Long-tailed Finch (Poephila acuticauda). Avian Research, 6: 1-10.

Støstad, H. N., Aldwinckle, P., Allan, A., Arnold, K. E. (2017). Foraging on human-derived foods by urban bird species. Bird Study, 64: 178-186.

Støstad, H. N., Johnsen, A., Rowe, M. Lifjeld, J. T. (2018a). Sperm head abnormalities are more frequent in songbirds with more helical sperm. Unpublished manuscript.

Støstad, H. N., Johnsen, A. Lifjeld, J. T. Rowe, M. (2018b). Sperm head morphology is associated with sperm swimming speed: A comparative study of songbirds using electron microscopy. Evolution, in press. doi:10.1111/evo.13555.

Surai, P., Blesbois, E., Grasseau, I., Chalah, T., Brillard, J.-P., Wishart, G., Cerolini, S., Sparks, N. (1998). Fatty acid composition, glutathione peroxidase and superoxide dismutase activity and total antioxidant activity of avian semen. Comparative Biochemistry and Physiology Part B: Biochemistry and Molecular Biology, 120: 527-533.

Surai, P., Noble, R., Sparks, N., Speake, B. (2000). Effect of long-term supplementation with arachidonic or docosahexaenoic acids on sperm production in the broiler chicken. Journal of Reproduction and Fertility, 120: 257-264.

Tomášek, O., Albrechtová, J., Němcová, M., Opatová, P., Albrecht, T. (2017). Trade-off between carotenoidbased sexual ornamentation and sperm resistance to oxidative challenge. Proceedings of the Royal Society B, 284: 20162444.

Tryjanowski, P., Skórka, P., Sparks, T.H., Biaduń, W., Brauze, T., Hetmański, T., Martyka, R., Indykiewicz, P., Myczko, Ł., Kunysz, P., Kawa, P. (2015). Urban and rural habitats differ in number and type of bird feeders and in bird species consuming supplementary food. Environmental Science and Pollution Research, 22: 15097-103.

Twining, C. W., Brenna, J. T., Hairston, N. G., Flecker, A. S. (2016a). Highly unsaturated fatty acids in nature: what we know and what we need to learn. Oikos, 125: 749-760.

Twining, C. W., Brenna, J. T., Lawrence, P., Shipley, J. R., Tollefson, T. N., Winkler, D. W. (2016b). Omega-3 longchain polyunsaturated fatty acids support aerial insectivore performance more than food quantity. Proceedings of the National Academy of Sciences, 113: 10920-10925.

Wilson, H., Piesco, N., Miller, E., Nesbeth, W. (1979). Prediction of the fertility potential of broiler breeder males. World's Poultry Science Journal, 35: 95-118.

Wolfson, A. (1952) The Cloacal Protuberance: A means for determining breeding condition in live male passerines. Bird-Banding, 23: 159-65.

Zanini, S. F., Torres, C., Bragagnolo, N., Turatti, J. M., Silva, M. G., Zanini, M. (2003). Evaluation of the ratio of $\omega 6: \omega 3$ fatty acids and vitamin $E$ levels in the diet on the reproductive performance of cockerels. Archives of Animal Nutrition, 57: 429-442. 